\title{
Silicon Photomultipliers (SiPM) as novel photodetectors for PET
}

\author{
Alberto Del Guerra ${ }^{\mathrm{a}, *}$, Nicola Belcari ${ }^{\mathrm{a}}$, Maria Giuseppina Bisogni ${ }^{\mathrm{a}}$, Francesco Corsi ${ }^{\mathrm{c}}$, Maurizio Foresta $^{\mathrm{c}}$, \\ Pedro Guerra ${ }^{\mathrm{d}}$, Sara Marcatili ${ }^{\mathrm{b}}$, Andres Santos ${ }^{\mathrm{d}}$, Giancarlo Sportelli ${ }^{\mathrm{d}}$ \\ a Department of Physics "E. Fermi", University of Pisa and INFN, Sezione di Pisa, I-56127 Pisa, Italy \\ ${ }^{b}$ INFN, Sezione di Pisa, I-56127 Pisa, Italy \\ 'Department of Electronic Engineering, Politecnico di Bari and INFN Sezione di Bari, I-70125 Bari, Italy \\ 'BIT, Universidad Politecnica de Madrid, S-28040 Madrid, Spain
}

A R T I C L E I N F O

Keywords:

SiPM

PET

Photodetectors

\begin{abstract}
A B S T R A C T
Next generation PET scanners should fulfill very high requirements in terms of spatial, energy and timing resolution. Modern scanner performances are inherently limited by the use of standard photomultiplier tubes. The use of Silicon Photomultipliers (SiPMs) is proposed for the construction of a 4D-PET module of $4.8 \times 4.8 \mathrm{~cm}^{2}$ aimed to replace the standard PMT based PET block detector. The module will be based on a LYSO continuous crystal read on two faces by Silicon Photomultipliers. A high granularity detection surface made by SiPM matrices of $1.5 \mathrm{~mm}$ pitch will be used for the $x-y$ photon hit position determination with submillimetric accuracy, while a low granularity surface constituted by $16 \mathrm{~mm}^{2}$ SiPM pixels will provide the fast timing information ( $t$ ) that will be used to implement the Time of Flight technique (TOF). The spatial information collected by the two detector layers will be combined in order to measure the Depth of Interaction (DOI) of each event ( $z$ ).

The use of large area multi-pixel Silicon Photomultiplier (SiPM) detectors requires the development of a multichannel Data Acquisition system (DAQ) as well as of a dedicated front-end in order not to degrade the intrinsic detector capabilities and to manage many channels. The paper describes the progress made on the development of the proof of principle module under construction at the University of Pisa.
\end{abstract}

\section{Innovative PET photodetectors}

Advance in PET detectors has always been moved by the goal of obtaining functional and spatial information as accurate as possible, while maintaining a low dose to the patient [1-3].

The seek for the optimum detector involves the improvement of different aspects. The increase in sensitivity is of primary importance in order to reduce the dose. For this reason, the ideal photodetector should present high intrinsic photodetection efficiency and should be versatile so as to be easily arranged in scanners with a good angular coverage. Also the use of a high granularity photodetector is advisable since it allows obtaining a high spatial resolution. However, it has to be taken into account that reducing the pixel size could result in lowering the sensitivity. Moreover, a fast photodetector would consent to increase the Signal to Noise Ratio (SNR) by measuring the Time of Flight (TOF) difference of the two annihilation photons [4].

\footnotetext{
${ }^{*}$ Corresponding author. Tel.: +39050 2214942; fax: +390502214333.

E-mail addresses: alberto.delguerra@df.unipi.it (A. Del Guerra), nicola.belcari@df.unipi.it (N. Belcari), bisogni@pi.infn.it (M. Giuseppina Bisogni), corsi@poliba.it (F. Corsi), foresta@deemail.poliba.it (M. Foresta), pguerra@ciber-bbn.es (P. Guerra), sara.marcatili@pi.infn.it (S. Marcatili), andres@die.upm.es (A. Santos), gsportelli@die.upm.es (G. Sportelli).
}

In the last 20 years, PET scanners have highly improved their performances thanks to the great progresses made in the Photomultiplier tube technology and to the newly developed scintillator crystals. In fact, despite many assembly solutions having been investigated, the standard PET block detector basically still consists of photomultiplier tubes (PMTs) coupled to pixilated scintillator matrices [5].

These progresses have led to the increase in energy and spatial resolution capabilities and to an appreciable increment in the inherent detector sensitivity. However, further significant improvements are not conceivable maintaining the same technology. Despite the fact that their dimension has lowered in the last years, PMTs package is constrained by the vacuum technology; hence, they do not have a high packing fraction. Also, the implementation of the TOF technique is not straightforward because of their time resolution of hundreds of picoseconds.

But above all, further increase in the spatial resolution with the PMT solution requires the construction of crystal matrices with pixel size of the order of $1 \mathrm{~mm}$ and uniform scintillation properties: a procedure which is technologically challenging and definitely very expensive.

Since its poor capability to reveal detailed morphological structures is an intrinsic limitation of Positron Emission Tomography, the 
need for a better identification of anatomical contours has lead to the development of hybrid imaging systems composed by a PET scanner associate to an X-ray Computed Tomography (CT) apparatus [6]. Their use is nowadays widely diffused in day-to-day clinical practice, even if they could present the disadvantage of contributing to an increase in the total dose delivered to the patient. Thus, another possibility is currently being exploited, addressing the problem of an improved capability of structures determination. Magnetic Resonance Imaging (MRI), in addition to providing exceptionally high resolution images, is a functional imaging technique, which is able to investigate the phenomena complementary to those of interest in PET without resulting in an additional dose delivering [7-9]. But even this approach is hardly achievable using PMTs since they are inherently sensitive to magnetic fields.

Recently, many research groups have proposed the use of solid state photodetectors for PET applications $[10,11]$ in order to overcome all these limitations. In particular, the Silicon Photomultiplier (SiPM) has emerged as the ultimate candidate for the replacement of the standard PMT based solution [12,13].

\section{The Silicon Photomultiplier (SiPM)}

A SiPM [14,15] pixel is a matrix of the Geiger-mode avalanche photodiodes (microcells) connected in parallel by means of a resistive layer. In this way they permit to accurately measure the energy deposited for a moderate photon flux ( $\left.N_{\text {photons }} \ll N_{\text {microcells }}\right)$. In order to obtain an accurate measurement of the spatial distribution of the incident radiation as well, they can be arranged in arrays with a pitch down to $1.5 \mathrm{~mm}$ with a negligible dead area [16,17]. Hence, the use of SiPM will allow covering large detection surfaces with a high granularity at low cost. In fact, since they present an intrinsic high spatial resolution, they can be coupled to monolithic scintillator crystals, reducing the overall cost of the detector and avoiding the loss of efficiency of finely pixilated crystals.

Moreover, these devices have reached a photon detection efficiency comparable to that of standard PMTs (up to 30\%) thanks to the extensive process of optimization [15] addressed to enhance the quantum efficiency in the region of emission of most scintillators used in PET (around $420 \mathrm{~nm}$ ).

Their very good intrinsic time jitter (about 70 ps FWHM at single photoelectron level in the case of SiPM pixels) makes viable the realization of human TOF-PET scanners if a very fast scintillator is adopted [18]. In fact, if the contribution of the photodetector can be neglected, as in the case of SiPMs, PET detector timing resolution is only limited by the scintillator crystal decay response. It has been demonstrated [19] that by coupling the SiPM pixels to LYSO crystals of the same dimension and reading them in time coincidence, the time jitter at $511 \mathrm{keV}$ is slightly above the $100 \mathrm{ps}$ (sigma).

Furthermore, the high intrinsic gain (up to $10^{6}$ ) obtainable at low bias voltage $(\sim 50 \mathrm{~V})$, and the consequent high Signal to Noise Ratio makes it redundant the use of sophisticated electronics.

Eventually, as it is a solid state detector, SiPM is intrinsically magnetic field insensitive, and hence can be used in the construction of combined PET/MRI systems. This possibility has already been explored by means of extensive tests in a magnetic resonance environment of $1.5 \mathrm{~T}$. SiPM capabilities in terms of energy resolution at $511 \mathrm{keV}$, and single photon resolution have been measured inside a MR bore with the gradients switched on and compared to those obtained on the bench. Results showed an excellent agreement between the two sets of data [20].

In the last years, its performances as PET photodetector has been deeply investigate in our research group. SiPM matrices have been coupled to scintillator of different geometries and types and tested by irradiating them with a ${ }^{22} \mathrm{Na}$ source.
Table 1

Main characteristics of the Silicon Photomultiplier as photodetector for PET applications

\begin{tabular}{ll} 
Energy resolution & $\sim 15 \%$ FWHM at $511 \mathrm{keV}$ \\
Spatial resolution coupled to an LSO slab & $0.9 \mathrm{~mm}$ FWHM \\
Intrinsic timing resolution & $\begin{array}{l}70 \text { ps sigma at single } \\
\text { photoelectron level }\end{array}$ \\
$\begin{array}{l}\text { Timing resolution (in coincidence) coupled } \\
\text { to an LSO slab }\end{array}$ & 120 ps sigma at $511 \mathrm{keV}$ \\
\hline
\end{tabular}

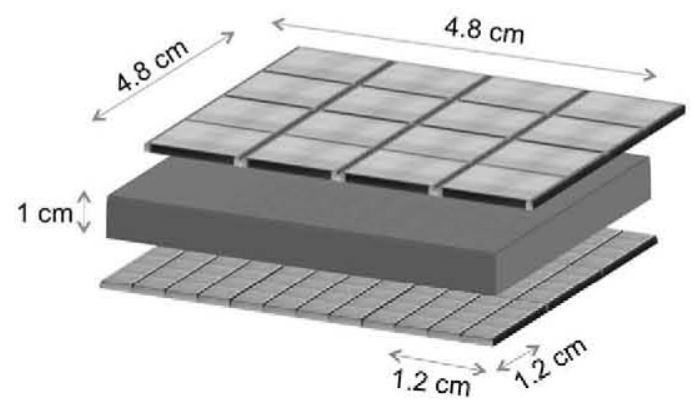

Fig. 1. Conceptual design of the 4D-PET prototype.

Results obtained with LSO continuous slab of the same size of the SiPM matrices are considered particularly interesting for our purposes; we measured a typical energy resolution of 15\% FWHM at $511 \mathrm{keV}$ and above all a spatial resolution of $0.9 \mathrm{~mm}$ FWHM for the single device (SiPM matrix+LSO) $[16,17,21]$.

Table 1 shows the most interesting characteristics of the Silicon Photomultiplier as PET photodetector.

\section{Architecture of a novel SiPM based block detector}

At INFN Pisa we are developing a 4D-PET module featuring high spatial and time resolution capabilities. The module is conceived as a novel "block detector" in order to replace the building block of next generation of PET tomographs. The detector module is implemented with novel technologies such as fast scintillators, high granularity and fast photodetectors, integrated electronics, FPGA based read-out and DAQ electronics. The design of the module has been determined with the aim of achieving excellent 2D spatial resolution ( $X$ and $Y$ coordinates), Depth of Interaction measurement ( $Z$ coordinate) and fast timing properties (time coordinate) that will result in a superior performance far beyond the state of the art of the currently available PET detectors.

The proposed 4D-PET module is based on a continuous LSO:Ce scintillating crystal of $4.8 \times 4.8 \mathrm{~cm}^{2}$ size coupled on both sides to Silicon Photomultipliers (see Fig. 1).

On the side where the annihilation photons impinge (the front side), an array of large SiPMs collects the scintillation light providing the timing information and the trigger for the acquisition of the event. Since the photon collection statistics can be considered Poissonian, a relatively large $\left(4 \times 4 \mathrm{~mm}^{2}\right)$ single pixel area has been selected in order to increase the total number of photons collected and hence increasing the time resolution. On the other side (the back side), the same light flash is collected by a finely pixilated SiPM surface consisting of $4 \times 4$ matrices of 64 pixel each on a $1.5 \mathrm{~mm}$ pitch. This detection surface is intended to provide the hit position in the $X-Y$ coordinates with a submillimetric resolution. The spatial information provided by both detection surfaces will be combined and used to reconstruct the Depth of Interaction (DOI) of each event so as to correct for the parallax error [22]. The cluster of pixels activated by the event provides also the energy released in the crystal. The module conceptual design is illustrated in Fig. 1. 


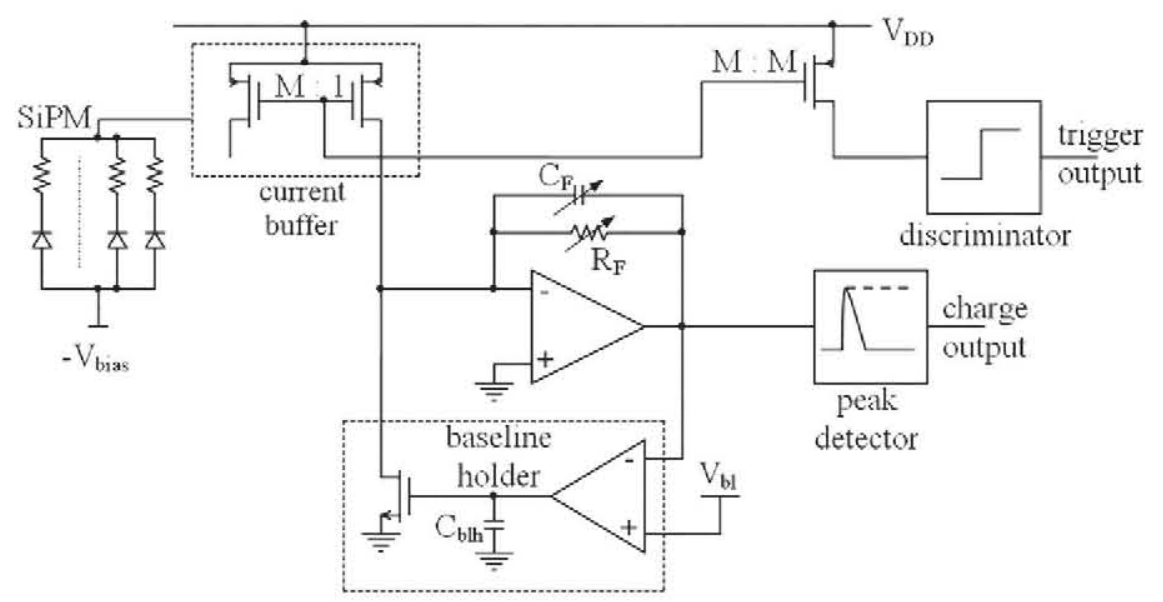

Fig. 2. Circuit schematic of one analog channel of the BASIC.

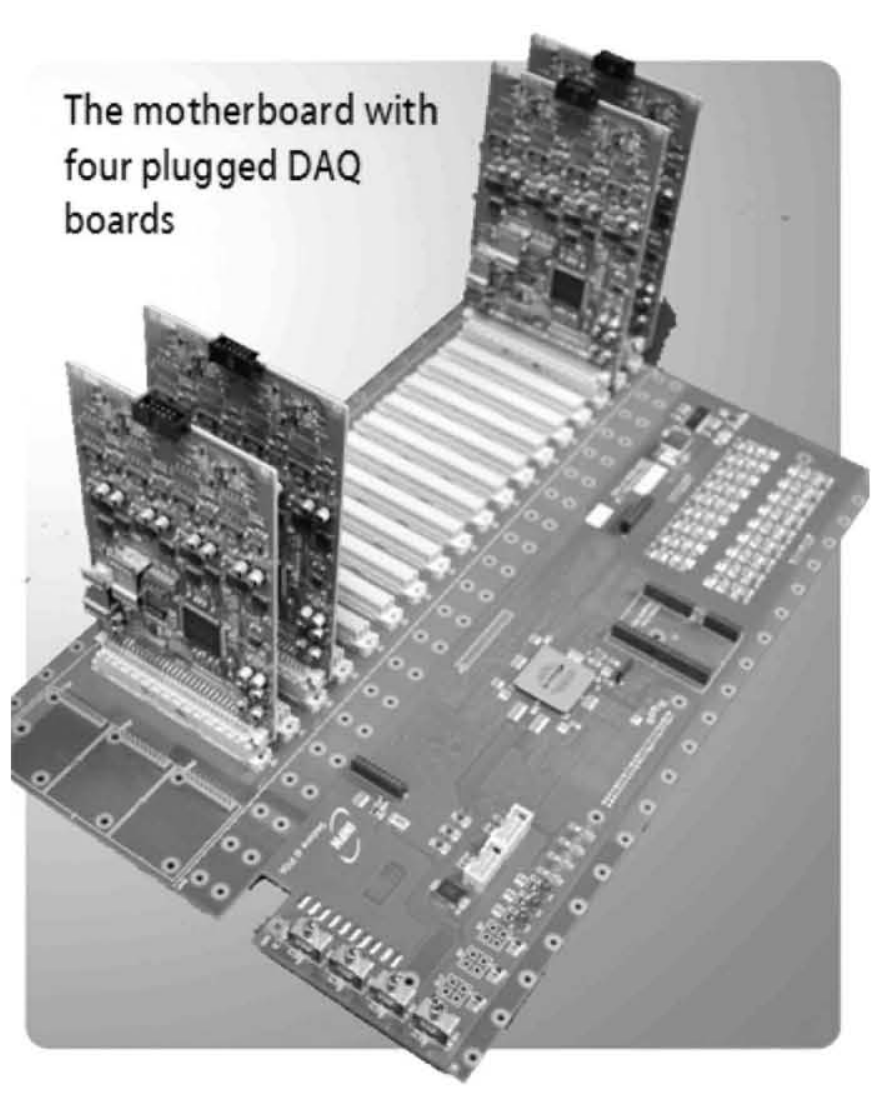

Fig. 3. The data acquisition system. The mother board with 4 DAQ boards plugged is shown.

\section{A dedicated read-out system}

A smaller module prototype is at the moment under construction at our laboratories, having a detection surface equal to one quarter of the final device described in the previous paragraph. In order to manage the many channels of the module (256 on the high granularity surface and 36 on the low granularity one) a dedicated acquisition system has been designed and produced. Also a dedicated front-end is required mainly because SiPM huge gain (up to $10^{6}$ ) typically causes the saturation of front-end chips developed for other photodetectors.

The read-out system, which is currently being developed, is based on an ASIC chip that handles both the signals pre-amplification and the fast-or trigger generation and on a dedicated acquisition system controlled by a high performances FPGA, details of which are provided in the next paragraph.

\subsection{The front-end ASIC}

The use of SiPM matrices makes it necessary to develop a read-out system capable to manage signals from many channels. This system should also exhibit excellent timing properties (time jitter below few hundreds of ps) and good spectroscopy capabilities not to degrade SiPM intrinsic performances. The data acquisition should sustain a $10 \mathrm{kHz} / \mathrm{cm}^{2}$ rate, which is the maximum expected in a pre-clinical scenario for a small animal PET scanner. The front-end should be based on Application Specific Integrated Circuit (ASIC), so as to manage many channels and keep the system compact at the same time. Standard ASIC chips are not ideal for SiPM applications because they have been mainly conceived for PMTs, and, when connected to a SiPM, they generally saturate because of its huge intrinsic gain. Hence, a dedicated front-end should be designed, since SiPM matrices are still novel detectors.

The signals coming from the $8 \times 8$ pixels of one matrix will be independently acquired by 2 custom 32 channel front-end ASIC (the BASIC chip) [23], developed at Polytechnic of Bari in submicron CMOS technology.

Each front-end channel (see Fig. 2) consists of a current amplifier reading on a very low impedance input node (virtual ground) the current signal delivered by the detector. The current mirror at the input allows the splitting of the signal in two branches: one is used to send the output current to a current discriminator, which extracts the trigger signal associated to the timing of the event, while the other is sent to an integrator proving a variable gain from 0.33 to $1 \mathrm{~V} / \mathrm{pC}$, in order to obtain a voltage proportional to the charge delivered by the event. The maximum dynamic range obtainable at lower gain is about $70 \mathrm{pC}$, which is enough to read $511 \mathrm{keV}$ photons signals avoiding saturation problems.

A current peak detector is used to hold the peak value of this current and to perform the conversion by means of an external ADC. The output of the $8 / 32$ fast triggers are sent to an internal OR circuit in order to generate the trigger (referred as FAST-OR) for the read-out of the whole chip. The 8 channel version of the ASIC has already been produced and is currently being tested, while a 32 channel version will be shortly available and it will be implemented in the final acquisition system.

\subsection{The acquisition board}

A modular acquisition system has been developed at the University of Pisa in order to manage the many channels of two 4D-PET modules and to read them in time coincidence. 


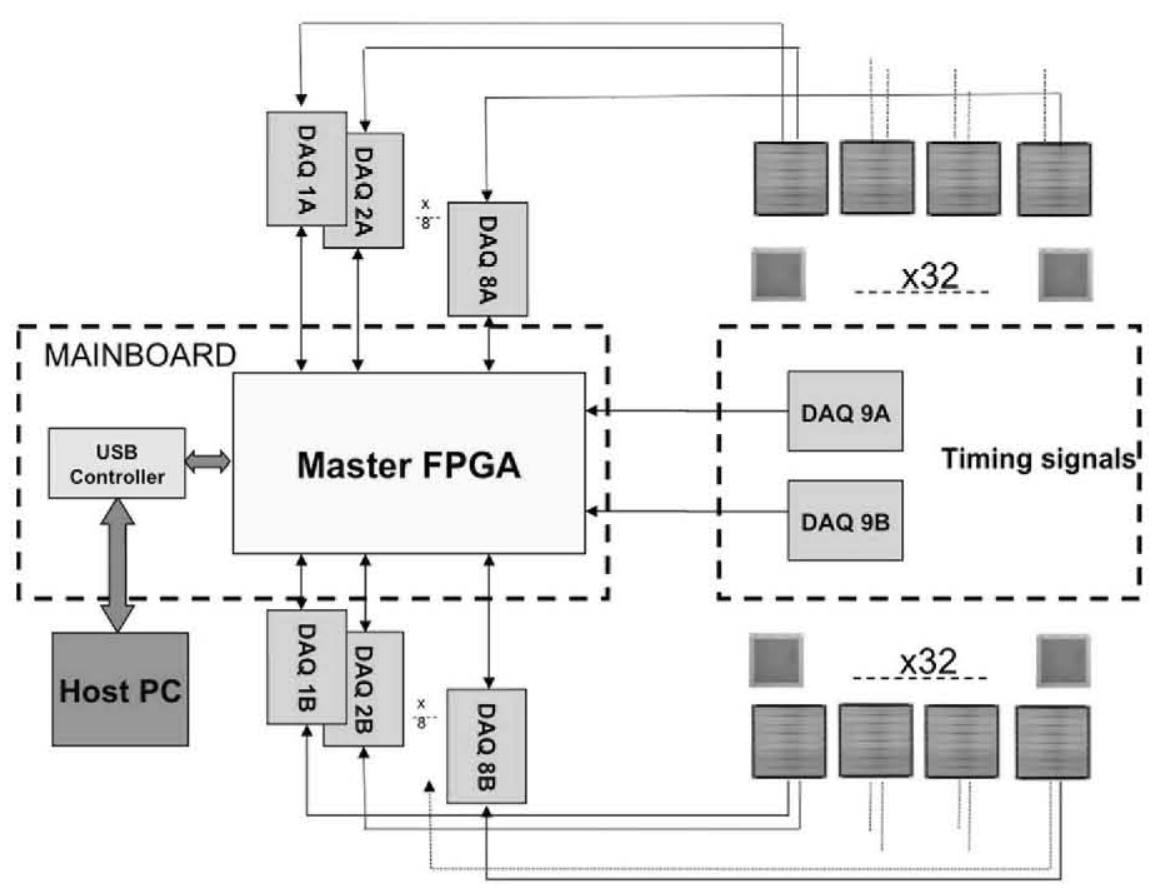

Fig. 4. Acquisition architecture for the 4D-PET block detector.

The core of the system is a cross-application acquisition board (called mother board) capable of handling up to 18 Data Acquisition (DAQ) boards, each one housing one front-end chip (see Fig. 3) [24]. Hence, each DAQ board will read from 8 to 32 channels of the detector module depending on the version of the ASIC mounted. The ASIC is housed on a mezzanine board in order to allow an easy replacement of the chip which is possible thanks to the ASIC serial output. In each DAQ board, a single channel, 10 bit, 105 MSPS ADC allows for the conversion of the signals coming from the sample and hold circuit in the ASIC; an FPGA (Cyclone II from Altera) on each DAQ controls the data read-out and sends the energy and timing signals to the mother board when it receives a valid trigger. A high performance FPGA (Stratix III from Altera) on the mother board handles the transmission and synchronization of the trigger and controls the signals and the acquisition and storage of the data in a host PC.

A fully digital time coincidence algorithm with a time window of about $7 \mathrm{~ns}$ FWHM is implemented on the Stratix III. The possibility of implementing a digital channel TDC in the mother board FPGA as well has been investigated.

Fig. 4 describes the acquisition architecture mounting the 32 channels version of the ASIC. The system can manage the read-out of two modules of $256+32$ channel each. In fact, 8 DAQ boards can be used for the read-out of the 256 channels of the high granularity detection surface of each detector block, while one DAQ board can be used for the read-out of the signals from the low granularity surface.

The FAST-OR signals from the DAQs are sent to the mother board to generate the trigger for the acquisition of the data from the whole module and to evaluate the coincidence.

\section{Conclusions}

SiPM matrices have been demonstrated to be optimum candidates for the next generation of PET apparatuses. Their intrinsic features allow for the construction of PET systems with performances far better than those of the modern scanners. A 4D-PET module is under development at the University of Pisa with the aim to replace the standard PMT based PET block detector. It will provide DOI and TOF information and a submillimetric spatial resolution in the $X-Y$ coordinates.

Currently the data acquisition system is available and completely debugged, and most of the firmware has been written. First tests on the DAQ boards mounting the BASIC chip are ongoing and we are performing a full characterization of all the logic functions implemented on the system and an accurate calibration of the analog electronics. Afterwards we will connect the SiPM matrices to the read-out system in order to validate the feasibility of the 4D-PET module proposed.

\section{Acknowledgements}

This work has been supported by the INFN-Group V (National Institute of Nuclear Physics), by Italian MIUR-PRIN 2007 under the project: "A very high spatial resolution small animal PET scanner based on high granularity Silicon Photomultipliers", and by the European Community in the framework of the FP7 ENVISION project.

\section{References}

[1] H. Uchida, et al., Trans. Nucl. Sci. NS33 (1) (1986) 464

[2] D. Grigoriev, et al., Trans. Nucl. Sci. NS44 (1997) 990.

[3] R. Scafè, et al., Nucl. Instr. and Meth. A 513 (2003) 36.

[4] W.W. Moses, Trans. Nucl. Sci. NS50 (5) (2003) 1325.

[5] M.E. Casey, R. Nutt, Trans. Nucl. Sci. NS33 (1) (1986) 460.

[6] D.W. Townsend, et al., Br. J. Radiol. 75 (2002) S24.

[7] B.J. Pichler, et al., J. Nucl. Med. 47 (2006) 639.

[8] G. Lucignani, Eur. J. Nucl. Med. Mol. Imaging 33 (2006) 969.

[9] P.K. Marsden, et al., Br. J. Radiol. 72 (2002) $\$ 53$.

[10] B. Pichler, et al., Trans. Nucl. Sci. NS45 (3) (1998) 1298.

[11] A.N. Otte, et al., Nucl. Instr. and Meth. A 545 (2005) 705.

[12] S.I. Ziegler, Eur. J. Nucl. Med. 28 (2001) 136.

[13] S. Moehrs, et al., Phys. Med. Biol (2006) 1113.

[14] V. Saveliev, V. Golovin, Nucl. Instr. and Meth. A 442 (2000) 223.

[15] C. Piemonte, Nucl. Instr. and Meth. A 568 (2006) 224.

[16] G. Llosa, et al., Trans. Nucl. Sci. NS56 (3) (2009) 543.

[17] G. Llosa, et al., Trans. Nucl. Sci. NS56 (5) (2009) 2586.

[18] G. Collazuol, et al., Nucl. Instr. and Meth. A 581 (2007) 461.

[19] M.G. Bisogni, et al., Nucl. Instr. and Meth. A(2010). doi:10.1016/j.nima.2010.07.016.

[20] R. Hawkes et al., M18-118, Conference Record of the 2007 IEEE NSS-MIC.

[21] G. Llosa et al., M05-91, Conference Record of the 2009 IEEE NSS-MIC.

[22] W.W. Moses, S.E. Derenzo, Trans. Nucl. Sci. NS41 (1994) 1441.

[23] F. Corsi et al., N19-2, Conference Record of the 2009 IEEE NSS-MIC

[24] G. Sportelli et al., M09-332, Conference Record of the 2009 IEEE NSS-MIC. 